\title{
LOW- $\Gamma$ JETS FROM COMPACT STELLAR MERGERS: CANDIDATE ELECTROMAGNETIC COUNTERPARTS TO GRAVITATIONAL WAVE SOURCES
}

\author{
Gavin P Lamb and Shino Kobayashi \\ Astrophysics Research Institute, Liverpool John Moores University, IC2, Liverpool Science Park, 146 Brownlow Hill, Liverpool L3 5RF, UK \\ Received 2016 May 9; revised 2016 July 22; accepted 2016 July 22; published 2016 September 28
}

\begin{abstract}
Short gamma-ray bursts (GRBs) are believed to be produced by relativistic jets from mergers of neutron stars (NSs) or NSs and black-holes (BHs). If the Lorentz-factors $\Gamma$ of jets from compact stellar mergers follow a similar power-law distribution to those observed for other high-energy astrophysical phenomena (e.g., blazars, active galactic nuclei), the population of jets should be dominated by low- $\Gamma$ outflows. These jets will not produce prompt gamma-rays, but jet energy will be released as X-ray/optical/radio transients when they collide with the ambient medium. Using Monte Carlo simulations, we study the properties of such transients. Approximately $78 \%$ of merger jets $<300 \mathrm{Mpc}$ result in failed GRBs if the jet $\Gamma$ follows a power-law distribution of index -1.75 . X-ray/ optical transients from failed GRBs will have broad distributions of their characteristics: light-curves peak $t_{p} \sim 0.1-10$ days after a merger; flux peaks for X-ray $10^{-6} \mathrm{mJy} \lesssim F_{x} \lesssim 10^{-2} \mathrm{mJy}$; and optical flux peaks at $14 \lesssim m_{g} \lesssim 22$. X-ray transients are detectable by Swift XRT, and $\sim 85 \%$ of optical transients will be detectable by telescopes with limiting magnitude $m_{g} \gtrsim 21$, for well localized sources on the sky. X-ray/optical transients are followed by radio transients with peak times narrowly clustered around $t_{p} \sim 10$ days, and peak flux of $\sim 10-100 \mathrm{mJy}$ at $10 \mathrm{GHz}$ and $\sim 0.1 \mathrm{mJy}$ at $150 \mathrm{MHz}$. By considering the all-sky rate of short GRBs within the LIGO/Virgo range, the rate of on-axis orphan afterglows from failed GRBs should be 2.6(26) per year for NS-NS (NS-BH) mergers, respectively. Since merger jets from gravitational-wave (GW) trigger events tend to be directed to us, a significant fraction of $\mathrm{GW}$ events could be associated with the on-axis orphan afterglow.
\end{abstract}

Key words: gamma-ray burst: general - gravitational waves

\section{INTRODUCTION}

Gamma-ray bursts (GRBs) are instantaneously the most luminous objects in the universe, produced by the deceleration of ultra-relativistic outflow (Lorentz factors $\Gamma \gtrsim 100$ ). The core-collapses of massive stars are the progenitors of long GRBs, and the mergers of binary compact stellar objects such as neutron stars (NSs) and black holes (BHs) are the possible progenitors of short GRBs (Woosley \& Bloom 2006; Nakar 2007; Berger 2014). In both cases accretion onto a compact object is likely to power the relativistic outflow and the same physical processes are involved. The outflow energy is first dissipated by internal shocks (or another form of internal dissipation) which produces prompt $\gamma$-rays. Later, the interaction of the outflow with the ambient medium produces an external shock which expands and produces the subsequent afterglow (e.g., Piran 2004; Zhang \& Mészáros 2004).

Relativistic motion is an essential ingredient in the GRB model although the exact outflow formation process is not known. Understanding the nature of the outflow, especially the acceleration, collimation, and energy content, is a major focus of international research efforts in the context of GRBs and other astrophysical jets. GRB outflows are conventionally assumed to be a baryonic jet (Paczyński 1986; Shemi \& Piran 1990), although polarization measurements imply that magnetic fields play a role in the jet acceleration (e.g., Götz et al. 2009; Steele et al. 2009; Yonetoku et al. 2011; Mundell et al. 2013). Relativistic outflows and possibly magnetic acceleration are features that GRBs, active galactic nuclei (AGNs), and microquasars have in common. Stellar tidal disruption by a massive $\mathrm{BH}$ is also likely to produce a relativistic jet (Bloom et al. 2011; Burrows et al. 2011; Levan et al. 2011; Zauderer et al. 2011; Cenko 2012). By studying and comparing the properties of these objects, we could gain an insight into the processes that govern the formation of relativistic jets (e.g., Marscher 2006a; Nemmen 2012).

In the case of blazars, we can measure apparent superluminal motion (i.e., lower limits of $\Gamma$ ), where reported apparent velocities are as high as $40-50 c$ for $\gamma$-ray bright blazars (Jorstad et al. 2005; Lister et al. 2009; Piner et al. 2012; Liodakis \& Pavlidou 2015). The Lorentz factor for AGNs is typically $1<\Gamma \leqslant 40$ (e.g., Marscher 2006a; Saikia et al. 2016, etc.) or $1<\Gamma \leqslant 50$ (Lister et al. 2009). Blazars with a high $\Gamma$ overpopulate centimeter-wave surveys of bright flat-spectrum sources because of beaming bias. Alternatively, a volumelimited sample of radio-loud AGNs would be dominated by objects with more mundane jets. A power-law distribution of Lorentz factors for AGNs can be assumed, $N(\Gamma) \sim \Gamma^{-a}$, where population synthesis studies show that a value of $a$ between 1.5 and 1.75 provides a good match between a synthetic and observed distribution of apparent velocities (Lister \& Marscher 1997; Marscher 2006b). Recent work indicates a value of $a=2.1 \pm 0.4$ for blazars (Saikia et al. 2016).

Many observations indicate that GRBs are produced by ultra-relativistic outflows with $\Gamma \gtrsim 100$. However, GRB progenitors might not always eject such a high- $\Gamma$ flow. For example, if the outflow is baryonic, the baryon loading might not always be optimal, resulting in lower Lorentz factors. For an outflow with low $\Gamma$, the internal dissipation processes (i.e., $\gamma$-ray production) happen when the outflow is still optically thick. Since we are currently discovering GRB events through wide field monitoring of the $\gamma$-ray sky (e.g., Swift, Fermi, IPN), a population of low $-\Gamma$ outflows might be undiscovered.

Compact stellar mergers are the most promising targets for ground-based gravitational wave (GW) detectors such as advanced LIGO, Virgo and KAGRA. The merger of a binary 
BH system produced the advanced LIGO detection GW150914, the first direct observation of GWs (Abbott et al. 2016b). Electromagnetic (EM) counterparts to BH-BH mergers are not expected; the FermiGamma-ray Burst Monitor (GBM) however, claimed a $2.9 \sigma$ detection of a weak $\gamma$-ray burst $0.4 \mathrm{~s}$ after the GW detection (Connaughton et al. 2016). If this burst was associated with GW150914 then an EM afterglow would also be present (Yamazaki et al. 2016). To maximize the science returns from further GW detections, the identification of an EM counterpart will be crucial. The $\gamma$-ray emission from short GRBs are an ideal EM counterpart to NSNS/NS-BH mergers, and potentially $\mathrm{BH}-\mathrm{BH}$ mergers. However, they occur relatively rarely within the range of $\mathrm{GW}$ detectors (300 Mpc for face-on NS-NS mergers); this is possibly because $\gamma$-ray emission is highly collimated, or the mis-match between short GRB peak energies and the Swift detection band can make detection more difficult. Additionally the intrinsic rate of compact object mergers within this volume is relatively low. More isotropic EM components such as macronovae are often discussed to localize a large sample of GW events (e.g., Nakar \& Piran 2011; Metzger \& Berger 2012; Gao et al. 2013; Kisaka et al. 2015).

In this paper, we discuss the possibility that a significant fraction of compact stellar mergers results in the production of low- $\Gamma$ jets $(\Gamma \lesssim 100)$. If such jets are common, X-ray, optical, and radio transients, i.e., on-axis orphan afterglows (Dermer et al. 2000; Huang et al. 2002; Nakar \& Piran 2002a; Rhoads 2003; Cenko et al. 2013, 2015), would be more frequent than short GRBs. Such low-frequency transients would accompany a good fraction of GW events and they allow for the accurate determination of the sky positions of the GW sources. The time lag between $\mathrm{GW}$ signals, where we can assume that the jet launch time $t_{0}$ is coincident with the merging time when the GW amplitude becomes maximal, and EM jet emission, will enable us to determine the $\Gamma$ distribution of jets from compact stellar mergers and it will provide another constraint on the acceleration process of relativistic jets. In Section 2 we discuss the background of relativistic motion in the standard GRB fireball model and the implications for prompt $\gamma$-ray emission. In Section 3 the case for a population of low Lorentz factor jets is made. Section 4 details the assumptions and conditions made by the Monte Carlo model plus the numerical results. Section 5 highlights the implications for $\mathrm{GW}$ rates within the LIGO/Virgo detection volume. In Section 6 conclusions are given.

\section{RELATIVISTIC MOTION AND THE PROMPT GAMMA- RAY EMISSION}

Observed GRBs contain a large fraction of high-energy $\gamma$-ray photons, which can produce electron-positron pairs if they interact with lower-energy photons. If the optical depth for this process is large, pairs will form rapidly and Compton scatter other photons, resulting in an increased optical depth. The optical depth for pair creation is very sensitive to the Lorentz factor of the source $\tau_{\gamma \gamma} \propto \Gamma^{-6}$ (e.g., Piran 1999; Lithwick \& Sari 2001 for the typical high-energy spectral index $\beta \sim 1$ ). The source becomes optically thin if it is expanding with a Lorentz factor $\Gamma \gtrsim 100$.

If there are baryons in GRB outflows, another limit on $\Gamma$ can be obtained by considering the scattering of photons by electrons associated with these baryons (e.g., Lithwick \& Sari 2001). Note that high polarization results still suggest magnetized baryonic jets, rather than Poynting-flux dominated jets (Steele et al. 2009; Mundell et al. 2013). The optical depth due to these electrons at radius $R$ is $\tau=\sigma_{T} E /\left(4 \pi R^{2} m_{p} c^{2} \Gamma\right)$ where $\sigma_{T}$ is the Thomson cross-section, $E$ is the total isotropic explosion energy and $m_{p}$ proton mass. Outflows become optically thin at the photospheric radius,

$$
R_{p} \sim 6 \times 10^{13} E_{51}^{1 / 2} \Gamma_{1}^{-1 / 2} \mathrm{~cm},
$$

where $E_{51}=E / 10^{51} \mathrm{erg}$ and $\Gamma_{1}=\Gamma / 10$. On the other hand, the variability timescale $\delta t$ in GRBs constrains the radius from which the radiation is emitted,

$$
R_{d} \sim \Gamma^{2} c \delta t \sim 3 \times 10^{11} \delta t_{-1} \Gamma_{1}^{2} \mathrm{~cm},
$$

where $\delta t_{-1}=\delta t / 0.1 \mathrm{~s}$. Requiring $R_{d}>R_{p}$, we obtain $\Gamma \gtrsim 80 E_{51}^{1 / 5} \delta t_{-1}^{-2 / 5}$. For outflows with a small Lorentz factor $\Gamma \lesssim 100$, the internal dissipation happens when the outflow is still optically thick. The photons will remain trapped and the thermal energy will be converted back to the kinetic form (Kobayashi \& Sari 2001; Kobayashi et al. 2002), and the prompt $\gamma$-ray emission would be suppressed (i.e., failed GRBs).

Usually outflows are assumed to have a sub-relativistic temperature after the internal dissipation, and the internal energy density is comparable to the mass energy density $e_{\text {int }} \sim e_{\text {mass. }}$. If a significant fraction of the internal energy is converted to electron-positron pairs, the number density of the electrons and positrons $\lesssim e_{\text {int }} / m_{e} c^{2}$ could be larger by a factor of $\lesssim m_{p} / m_{e}$ than that of electrons that accompany baryons, where $m_{e}$ is the electron mass. A more detailed discussion (Lithwick \& Sari 2001) also shows that the scattering of photons by pair-created electrons and positrons is nearly always more important than that by electrons that accompany baryons. Since the lepton pairs create an effective photosphere further out than the baryonic one, the approximation in Equation (1) will provide conservative estimates when we discuss failed GRB rates in Sections 4 and 5.

\section{ON-AXIS ORPHAN AFTERGLOW}

Even if a jet does not have a velocity high enough to emit $\gamma$ rays, it eventually collides with the ambient medium to emit at lower frequencies. Such synchrotron shock radiation has been well studied in the context of GRB afterglows (e.g., Mészáros \& Rees 1992, 1997; Kobayashi et al. 1999; Sari \& Piran 1999).

Because of relativistic beaming, the radiation from a jet can be described by a spherical model when $\Gamma>1 / \theta_{j}$ where $\theta_{j}$ is the jet half-opening angle. We here consider a relativistic shell with an energy $E$ and an initial Lorentz factor $\Gamma$ expanding into the ISM with particle density $n$. The deceleration of the shell happens at

$$
t_{\mathrm{dec}} \sim 0.48 E_{51}^{1 / 3} n_{-1}^{-1 / 3} \Gamma_{1}^{-8 / 3} \text { days, }
$$

where $n_{-1}=n / 10^{-1}$ protons $\mathrm{cm}^{-3}$, and $t_{\mathrm{dec}}$ is measured in the GRB rest frame. The typical frequency and the spectral peak flux of the forward shock emission at the deceleration time $t_{\mathrm{dec}}$ are,

$$
\begin{gathered}
\nu_{m} \sim 5.3 \times 10^{11} \epsilon_{B,-2}^{1 / 2} \epsilon_{e,-1}^{2} n_{-1}^{1 / 2} \Gamma_{1}^{4} \mathrm{~Hz} \\
F_{\nu, \max } \sim 35 D_{27}^{-2} \epsilon_{B,-2}^{1 / 2} n_{-1}^{1 / 2} E_{51} \mathrm{mJy}
\end{gathered}
$$

(Sari et al. 1998; Granot \& Sari 2002) where $\epsilon_{B}$ and $\epsilon_{e}$ are the microscopic parameters, $\epsilon_{B,-2}=\epsilon_{B} / 10^{-2}, \epsilon_{e,-1}=\epsilon_{e} / 10^{-1}$, and 
$D_{27}=D / 10^{27} \mathrm{~cm}$ (i.e., the LIGO range for face-on NS-NS mergers). The optical emission, assumed to be between the peak frequency $\nu_{m}$ and the cooling frequency $\nu_{c}$, is expected to rise as $F_{\nu} \sim t^{3}$ and decay as $\sim t^{-1}$ after the peak $t=t_{\text {dec }}$.

Self-absorption can significantly reduce synchrotron shock emission at low frequencies. The upper limit can be approximated as blackbody flux for the forward shock temperature (e.g., Sari \& Piran 1999); the limit at $t_{\mathrm{dec}}$ is

$$
F_{B B, \nu} \sim 2.2 \times 10^{2} \epsilon_{e,-1} \nu_{10}^{2} \Gamma_{1}^{2} D_{27}^{-2}\left(\frac{R_{\perp}}{2.5 \times 10^{16} \mathrm{~cm}}\right)^{2} \mathrm{mJy},
$$

where $\nu_{10}=\nu / 10 \mathrm{GHz}$ and the observable blast-wave size $R_{\perp} \sim 2 c \Gamma t$. Equalizing the synchrotron emission and the blackbody limit, we obtain the self-absorption frequency $\nu_{a} \sim 1.5 \epsilon_{B,-2}^{1 / 5} \epsilon_{e,-1}^{-1} n_{-1}^{3 / 5} E_{51}^{1 / 5} \mathrm{GHz}$ at the deceleration time $t_{\mathrm{dec}}$. The self-absorption limit initially increases as $t^{1 / 2}$, and then steepens as $t^{5 / 4}$ after $\nu_{m}$ crosses the observational frequency $\nu$. Considering that the synchrotron flux at $\nu<\nu_{m}$ also increases as $t^{1 / 2}$, if $\nu<\nu_{a}$ at $t_{\mathrm{dec}}$, the synchrotron emission would be reduced by the self-absorption at least until the passage of $\nu_{m}$ through the observational band at $t_{m} \sim 110 \epsilon_{B,-2}^{1 / 3} \epsilon_{e,-1}^{4 / 3} E_{51}^{1 / 3}(\nu / 150 \mathrm{MHz})^{-2 / 3}$ days. If the jet break happens while the flux is still self-absorbed, the light curve becomes flat $F_{\nu<\nu_{a}} \sim$ constant (Sari et al. 1999). However, this estimate is obtained by assuming rapid lateral expansion (i.e., $R_{\perp}^{2} \propto t$ ). Recent studies show that the sideways expansion is rather slow, especially for mildly relativistic jets (Granot \& Piran 2012; van Eerten \& MacFadyen 2012). We will assume that the blast-wave emission starts to decay at the jet break,

$$
t_{j} \sim 13.5 E_{51}^{1 / 3} n_{-1}^{-1 / 3}\left(\frac{\theta_{j}}{20^{\circ}}\right)^{8 / 3} \text { days }
$$

even if it is in the self-absorption phase. At low frequencies $\nu \lesssim 1 \mathrm{GHz}$ and early times, forward shock emission would be affected by synchrotron self-absorption. However, currently most radio afterglow observations are carried out at higher frequencies (e.g., VLA $8.5 \mathrm{GHz}$ ) at which self-absorption is more important for the reverse shock emission.

Just before the deceleration time $t_{\mathrm{dec}}$, a reverse shock propagates through the jet and heats the original ejecta from the central engine. The reverse shock region contains energy comparable to that in the forward shock region. However, it has a lower temperature due to a higher mass (i.e., lower energy per particle). The shock temperature and the typical frequency are lower by a factor of $\sim \Gamma$ and $\sim \Gamma^{2}$ compared to those of the forward shock (e.g., Kobayashi \& Zhang 2003). Although reverse shocks in low- $\Gamma$ jets could emit photons in the radio band, the self-absorption limit is tighter due to the lower shock temperature; we find that the forward shock emission always dominates. Note that we rarely catch the reverse shock emission even for regular GRBs with detectable $\gamma$-ray emission. We will discuss only the forward shock (i.e., blast wave) emission in this paper.

\section{MONTE CARLO MODEL}

Using the estimates of Lorentz factors based on long GRB afterglow peak times, Hascoët et al. (2014) demonstrated that an apparent correlation between isotropic $\gamma$-ray luminosity $L_{\gamma}$ and Lorentz factor $\Gamma$ can be explained by a lack of bright bursts with low Lorentz factors. They also predicted the existence of on-axis orphan afterglows of long GRB events. We here extend their argument to short GRBs, and we apply their formalism to cosmological (i.e., $\gamma$-ray satellite range) and local (i.e., GW detector range) events to study the on-axis orphan afterglows of failed short GRBs (i.e., low- $\Gamma$ events). The following assumptions are made in our simple Monte Carlo simulation of a synthetic population of merger events:

1. The redshift for each event is randomly determined using a distribution with a constant time delay with respect to the star formation rate, where the peak rate is at $z=0.9$. The redshift limits of $0 \leqslant z \leqslant 3$ are used for the cosmological sample, and $0 \leqslant z \leqslant 0.07$ for local sample, i.e advanced LIGO/Virgo detectable range $D \sim 1.5 \times 200 \mathrm{Mpc}=300 \mathrm{Mpc}$ for NS-NS mergers where the factor of 1.5 accounts for the stronger $\mathrm{GW}$ signal from face-on mergers (Kochanek \& Piran 1993). We use the event rate per unit comoving volume for short GRBs obtained by Wanderman \& Piran (2015), which is a function of $z$ as

$$
R_{\mathrm{SGRB}}(z) \propto\left\{\begin{array}{ll}
e^{(z-0.9) / 0.39} & z \leqslant 0.9 \\
e^{-(z-0.9) / 0.26} & z>0.9
\end{array} .\right.
$$

Numerical results for the cosmological cases are insensitive to the value of the maximum $z$ as long as it is much larger than unity.

2. A power-law distribution of Lorentz factors $N(\Gamma) \propto \Gamma^{-a}$ is assumed with reasonable limits $3 \leqslant \Gamma \leqslant 10^{3}$. Motivated by AGN studies (e.g., Lister \& Marscher 1997; Marscher 2006b), we choose $a=1.75$ as our fiducial value and the cases of $a=1.5$ and 2 will be briefly discussed.

3. The isotropic $\gamma$-ray luminosity $L_{\gamma}$ is randomly generated in the limit $10^{50} \mathrm{erg} \mathrm{s}^{-1} \leqslant L_{\gamma} \leqslant 10^{53} \mathrm{erg} \mathrm{s}^{-1}$ where the limits come from observational constraints and the luminosity distribution follows the form obtained by Wanderman \& Piran (2015),

$$
\Phi\left(L_{\gamma}\right) \propto \begin{cases}L_{\gamma}^{-1} & L_{\gamma} \leqslant 2 \times 10^{52} \mathrm{erg} \mathrm{s}^{-1} \\ L_{\gamma}^{-2} & L_{\gamma}>2 \times 10^{52} \mathrm{erg} \mathrm{s}^{-1}\end{cases}
$$

where this luminosity function is logarithmic in the interval d $\log L_{\gamma}$.

For each event, the dissipation radius $R_{d}=\Gamma^{2} c \delta t$ is evaluated using a random $\Gamma$ and the typical pulse width in short GRB light curves $\delta t=0.1 \mathrm{~s}$ (Nakar \& Piran 2002b). $\gamma$-ray photons are assumed to be emitted at $R_{d}$ with a random $\gamma$-ray luminosity $L_{\gamma}$ or equivalently a random isotropic $\gamma$-ray energy $E_{\gamma}=L_{\gamma} T$ where $T$ is the duration of short GRBs. We assume $T=0.6 \mathrm{~s}$ for all bursts as this is the median value for a log-normal distribution of durations for short GRBs (Zhang et al. 2012). The spectral peak energy in the $\nu F_{\nu}$ spectrum is known to be correlated with $L_{\gamma}$ (Yonetoku et al. 2004; Ghirlanda et al. 2009). The correlation is consistent for both long and 
short GRBs (Zhang et al. 2012), and is given by

$$
E_{p} \sim 300\left(\frac{L_{\gamma}}{10^{52} \mathrm{erg} \mathrm{s}^{-1}}\right)^{2 / 5} \mathrm{keV} .
$$

The $\nu F_{\nu}$ spectrum is assumed to follow a broken power-law with a low-energy index (below $E_{p}$ ) of $1.5=(-\alpha+2)$, and a high-energy index of $-0.25=(-\beta+2)$, where $\alpha$ and $\beta$ are the photon number spectral indices. The mean index values for all GRBs are $\alpha=1$ and $\beta=2.5$ (Gruber et al. 2014) but as short GRBs are typically harder than average we use the values $\alpha=0.5$ and $\beta=2.25$. The spectral peak is normalized as the value integrated between $1 \mathrm{keV}$ and $10 \mathrm{MeV}$ giving $L_{\gamma}$. If the outflow is optically thin, all the photons released at $R_{d}$ are radiated away. The event is considered to be detectable if the photon flux at the detector in the Swift band $(15-150 \mathrm{keV})$ is $>0.2$ photons s$~^{-1} \mathrm{~cm}^{-2}$ (Band 2006). We take into account the redshift of the spectrum when the photon flux is evaluated.

If the optical depth at the dissipation radius $R_{d}$ is more than unity, or equivalently the photospheric radius $R_{p}=\sqrt{\sigma_{T} E / 4 \pi m_{p} c^{2} \Gamma}$ is larger than the dissipation radius, the $\gamma$-ray emission would be suppressed where $E=E_{\gamma} / \eta$ is the explosion energy and $\eta$ is the conversion efficiency from the explosion energy to $\gamma$-rays. We use $\eta=0.2$, this is consistent with theoretical predictions (Kobayashi et al. 1997) and the fiducial value used in other works (Liang et al. 2010; Ghirlanda et al. 2012). The $\gamma$-ray energy injected at $R_{d}$ is adiabatically cooled, and the photons decouple from the plasma at $R_{p}$. Assuming a sharp transition from the optically thick to thin regime (see Beloborodov 2011 for a discussion of the fuzzy photosphere), we use hydrodynamic scalings to estimate the cooling factor. The internal energy density (photon energy density) decays as $e \propto R^{-8 / 3}$ and the Lorentz factor is constant for the outflow with a sub-relativistic temperature (Piran et al. 1993). Considering that the internal energy in the outflow shell with width $\Delta$ is $L_{\gamma} \Delta / c \propto e R^{2} \Delta \Gamma^{2}$, the luminosity of photons released at $R_{d}$ is

$$
L_{\gamma}\left(R_{p}\right) \sim L_{\gamma}\left(\frac{R_{p}}{R_{d}}\right)^{-2 / 3},
$$

where we have assumed no shell spreading, $\Delta \sim$ constant. The spectral peak energy is similarly shifted as $E_{p}\left(R_{p}\right)=E_{p}\left(R_{p} / R_{d}\right)^{-2 / 3}$. The photons in the coupled plasma undergo pair production and Compton down-scattering that progressively thermalizes the distribution (Hascoët et al. 2014). The electron temperature at $R_{d}$ can be approximated by a blackbody temperature $\phi_{b b} \sim\left(L_{\gamma} / 4 \pi R_{d}^{2} \Gamma^{2} c a\right)^{1 / 4}$ where $a$ is the radiation constant. The optical depth at $R_{d}$ is given by $\tau_{d} \sim\left(R_{p} / R_{d}\right)^{2}$. The condition for efficient thermalization is $\tau_{d} \gtrsim m_{e} c^{2} / k_{B} \phi_{b b}$ (Pe'er et al. 2005; Thomson 2007) where $m_{e}$ is the mass of an electron and $k_{B}$ the Boltzmann constant. The peak energy $E_{p}$ for such a case is given by $3 k_{B} \phi_{b b}$, above which the distribution is exponentially suppressed. For simplicity we assume $E_{p} \equiv E_{\max }$. If $\tau_{d} \lesssim m_{e} c^{2} / k_{B} \phi_{b b}$, the photons are not efficiently thermalized. The distribution is then limited by the efficiency of pair production where the maximum energy is $E_{\max } \sim 511\left(\Gamma / \tau_{d}\right) \mathrm{keV}$. The distribution is cut-off above this energy.
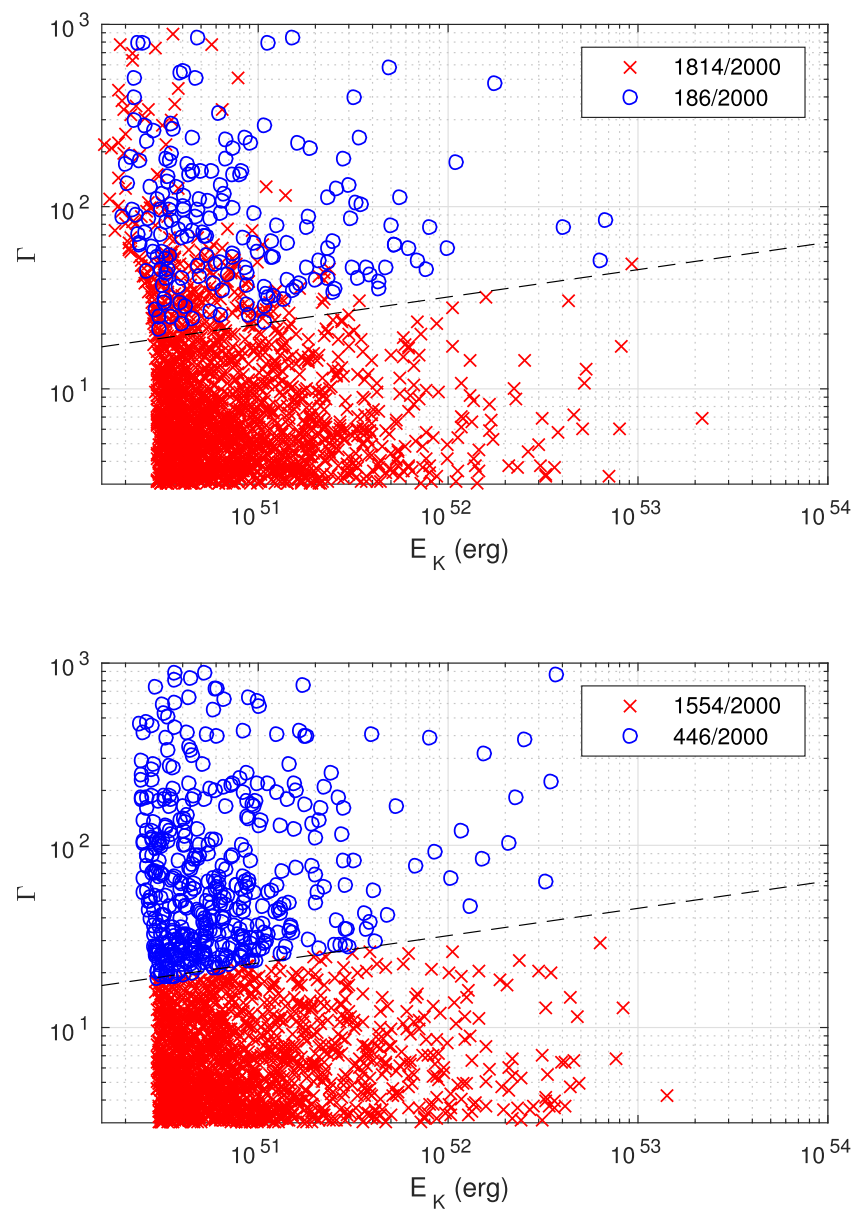

Figure 1. Isotropic kinetic energy $E_{\mathrm{K}}$ vs. bulk Lorentz factor $\Gamma$. Monte Carlo generated synthetic population of bursts. Top panel: cosmological sample of events with $0 \leqslant z \leqslant 3$. Bottom panel: local sample of events with $0 \leqslant z \leqslant 0.07$. Bursts with prompt emission flux above the Swift sensitivity are shown as the blue circles. Failed GRBs are indicated by the red crosses. $a=1.75$ is assumed.

\subsection{Numerical Results}

We generate a sample of $2 \times 10^{5}$ events and evaluate the $\gamma$ ray flux for each in the Swift band. To allow for clarity without losing the general trend, the results for a population of 2000 events are shown in Figure 1; the blue circles and red crosses show the events detectable and undetectable by Swift, respectively. The isotropic kinetic energy $E_{\mathrm{K}}$ is the energy in the blast wave after deceleration time, $E_{\mathrm{K}}=E-E_{\gamma}$, where $E$ is the total isotropic explosion energy, and $E_{\gamma}$ is the isotropic $\gamma$ ray energy at the photospheric radius $R_{p}$. The Lorentz factor $\Gamma$ of an outflow at $t<t_{\mathrm{dec}}$ is shown against this. The top panel shows the results with $0 \leqslant z \leqslant 3$, where we find a small fraction $\sim 9 \%$ of the total population and $\sim 49 \%$ of the events with $\Gamma>30$ are detectable by Swift. For the local population $0 \leqslant z \leqslant 0.07$, these fractions are higher, at $\sim 22 \%$ and $\sim 100 \%$ respectively, due to the proximity (see the bottom panel). The dashed line indicates the lower limit for a successful GRB; events below this line have the prompt $\gamma$-ray emission fully suppressed; the cut-off, with the parameters used, is given by $\Gamma \sim 16\left(E_{\mathrm{K}} / 10^{50} \mathrm{erg}\right)^{0.15}$.

In Figure 1, the low-energy limit of $E_{\mathrm{K}}$ is basically set by the Monte Carlo luminosity distribution (i.e., $L_{\gamma, \min }=10^{50}$ $\mathrm{erg} \mathrm{s}^{-1}$. Note that the explosion energy $E$ is higher than the 
$\gamma$-ray energy $L_{\gamma} T$ at the dissipation radius $R_{d}$ by a factor of $1 / \eta \sim 5$ ). If we consider the local population (the bottom panel), for the events above the dashed line (i.e., the blue circles) all of the $\gamma$-ray energy is successfully radiated away, whereas for the events below the dashed line (i.e., the red crosses), almost all of the $\gamma$-ray energy is reabsorbed into the outflow. Thus the distribution of $E_{\mathrm{K}}$ for the blue circles has a slightly lower limit. If we consider the cosmological population (the top panel), a fraction of events are distant and intrinsically dim. They are undetectable by Swift even if all gamma-ray energy is successfully radiated away at $R_{d}$. This is why there are red crosses above the dashed line for the cosmological population. The fraction of the events detectable by Swift weakly depends on $L_{\gamma, \min }$. If we assume $L_{\gamma, \min }=5 \times 10^{49}$ $\mathrm{erg} \mathrm{s}^{-1}$, Swift would be able to detect $\sim 6 \%$ of the total cosmological population, and $\sim 25 \%$ of the total local population.

Liang et al. (2010), Ghirlanda et al. (2012) and Tang et al. (2015) report correlations between Lorentz factor $\Gamma$ and the isotropic luminosity $L_{\gamma}$ (or the isotropic energy $E_{\gamma}$ ) for long GRBs: $E_{\gamma} \propto \Gamma^{4.00} ; L_{\gamma} \propto \Gamma^{2.15}$; and $L_{\gamma} \propto \Gamma^{1.92}$, respectively. However, such power-law relations could indicate a lower limit on $\Gamma$ for observable long GRBs with a given burst energy (Hascoët et al. 2014). In our simulation, we find that the detectable short bursts are always located above a line $\Gamma \sim 20\left(E_{\gamma} / 10^{49} \mathrm{erg}\right)^{0.17}$ giving a lower limit relation $E_{\gamma} \propto \Gamma^{5.88}$.

As discussed in Section 3, the kinetic energy $E_{\mathrm{K}}$ of the failed GRBs will be released as on-axis orphan afterglows at late times. Figure 2 shows the distributions of the peak flux (the top panel) and peak time (the bottom panel) of such X-ray, optical, and radio transients. To estimate these distributions, we have used the Monte Carlo results for the local sample $(D<300 \mathrm{Mpc})$ with model parameters: $n=10^{-1}$ protons $\mathrm{cm}^{-3}$ (Metzger \& Berger 2012; Berger 2014), $\epsilon_{B}=10^{-2}$, $\epsilon_{e}=10^{-1}$ (Panaitescu \& Kumar 2002; Yost et al. 2003; Berger 2014), the index of the power-law distribution of random electrons accelerated at shock $p=2.5$ (Sari et al. 1996; Daigne et al. 2011; Metzger \& Berger 2012), and the jet halfopening angle $\theta_{j}=20^{\circ}$ ensuring $t_{j}>t_{\mathrm{dec}}$ for our sample and is within the limits $16 \pm 10^{\circ}$ found by Fong et al. (2015) for short GRBs. The jet opening angle plays a role only when we estimate the jet break time.

The dotted green lines (Figure 2) indicate the distribution for $\mathrm{X}$-ray transients. The typical frequency of the blast wave emission $\nu_{m}$ is sensitive to the Lorentz factor $\nu_{m} \propto \Gamma^{4}$. Since for the local population the on-axis orphan afterglows are produced by low- $\Gamma$ jets $(\Gamma \lesssim 30)$, the typical frequency $\nu_{m}$ is expected to already be below the X-ray and optical band at the deceleration time $t_{\mathrm{dec}}$. The $\mathrm{X}$-ray and optical light curves should peak at $t_{\mathrm{dec}}$ and they have the same peak time distribution. Considering that the deceleration time $t_{\mathrm{dec}} \propto E_{\mathrm{K}}^{1 / 3} \Gamma^{-8 / 3}$ is mainly determined by $\Gamma$, we can roughly estimate the peak-time distribution $d N \propto \Gamma^{-a} d \Gamma \propto t_{\mathrm{dec}}^{3(a-1) / 8} d\left(\log t_{\mathrm{dec}}\right)$. For $a \gtrsim 1$, the distribution is wide and a large fraction of the events have the peaktime $t_{\mathrm{dec}}$ around several days after the merger event. If the minimum Lorentz factor $\Gamma_{\min }=2$ is assumed, the peak-time distribution would achieve the peak around a few weeks after the merger event. The distribution of the peak flux for X-ray, where the frequency is above the cooling frequency $\nu_{x}>\nu_{c}$, is $F_{p}=\left(\nu_{c} / \nu_{m}\right)^{-(p-1) / 2}\left(\nu_{x} / \nu_{c}\right)^{-p / 2} F_{\nu, \max } \propto \Gamma^{2(3 p-2) / 3} E_{\mathrm{K}}^{2 / 3}$,
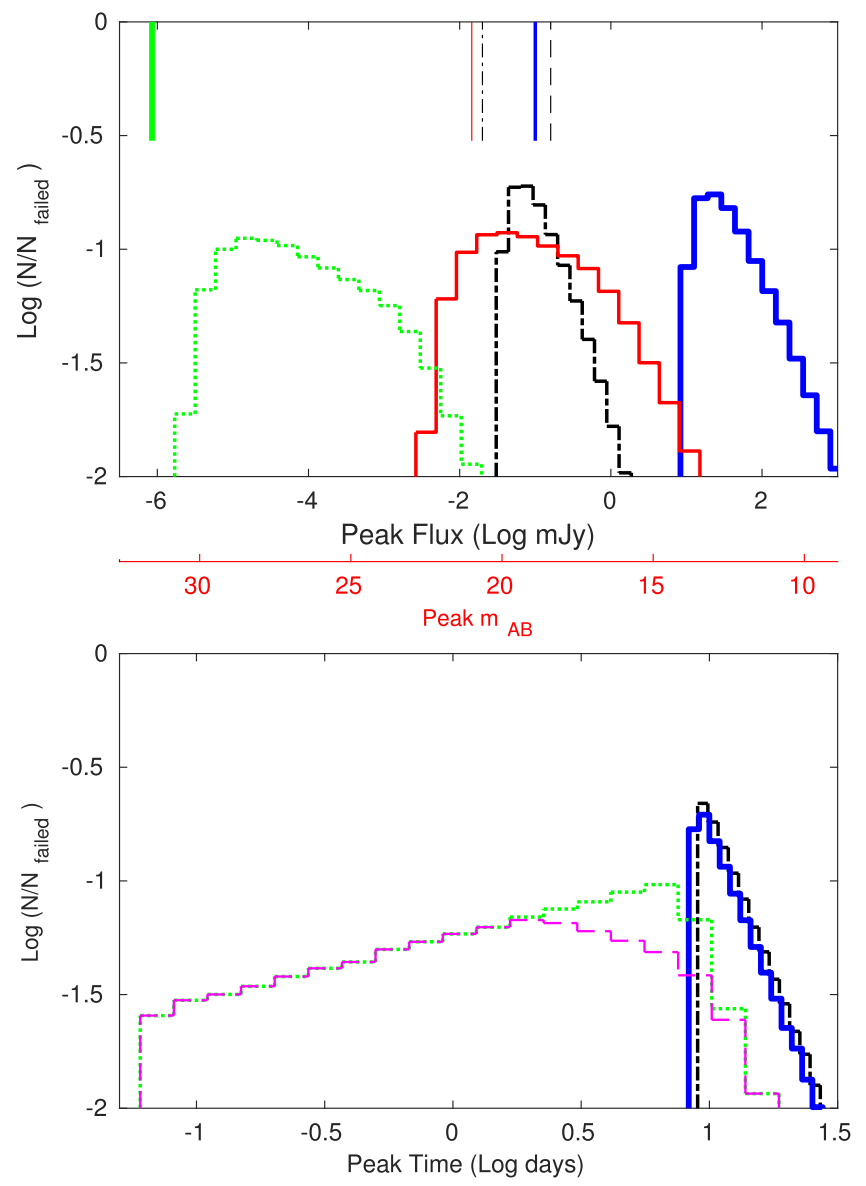

Figure 2. The peak-flux (top panel) and peak-time (bottom panel) distribution of on-axis orphan afterglows from failed GRB events within $300 \mathrm{Mpc}$. The distributions are normalized by the total number of failed GRBs. X-ray (dotted green line), optical (thick solid red line), radio $10 \mathrm{GHz}$ (thick solid blue line) and radio $150 \mathrm{MHz}$ (thick dashed-dotted black line). The vertical lines in the top panel indicate the sensitivity limits of telescopes (thick green XRT, thin red optical $2 \mathrm{~m}$, dashed-dotted SKA1-Low, and dashed 48 LOFAR), and the dashed magenta line in the bottom panel shows the distribution of bright events $m_{g} \leqslant 21$ (see the main text for details).

shown in the top panel. Given good localization, all of the $\mathrm{X}$-ray peak afterglow flux is above the minimum senstivity of the Swift XRT $2.4 \times 10^{-14} \mathrm{erg} \mathrm{cm}^{-2} \mathrm{~s}^{-1}$ for $10^{4} \mathrm{~s}$ (the vertical green thick solid line). The X-ray afterglows are below the trigger sensitivities of Swift BAT and MAXI, and too faint to be detectable by the Swift BAT survey.

The solid red line in the top panel and the dotted green line in the bottom panel indicate the distribution for optical ( $g$-band) transients. The $\mathrm{AB}$ magnitude $\mathrm{m}_{\mathrm{AB}}$ axis is added in the top panel to indicate the optical flux. For optical transients, peak flux is $F_{p}=\left(\nu_{\mathrm{opt}} / \nu_{m}\right)^{-(p-1) / 2} F_{\nu, \max } \propto \Gamma^{2(p-1)} E_{\mathrm{K}}$, and $85 \%$ of the optical orphan afterglows are brighter than $m_{g}=21$ (the vertical solid red line indicates this typical limit for mid-sized ( $\sim 2 \mathrm{~m}$ ) telescopes). The peak-time distribution for the bright events $\left(m_{g}<21\right)$ is shown as the the dashed magenta line in the bottom panel. The difference between the dotted green (representing both X-ray and optical in peak time) and dashed magenta line corresponds to the dim event population $\left(m_{g}>21\right)$. Since these events tend to have low- $\Gamma$, their typical frequencies are much lower than the optical band, and they peak at late times. 
The solid blue lines give the distribution for radio $(10 \mathrm{GHz})$ transients. The typical frequency $\nu_{m}$ is expected to be above $10 \mathrm{GHz}$ at the deceleration time $t_{\mathrm{dec}}$. The light curve peaks when the typical frequency $\nu_{m} \propto t^{-3 / 2}$ crosses the observational band: $t_{p} \propto E_{\mathrm{K}}^{1 / 3}$. Since the dynamics of the blast wave at $t>t_{\mathrm{dec}}$ depends only on the Sedov length $\propto E_{\mathrm{K}}^{1 / 3}$ and not on the initial Lorentz factor $\Gamma$, the peak-time distribution should be narrowly clustered, compared to the distribution of the optical transients. The Monte Carlo results actually give a narrow peak around $t_{p} \sim 10$ days. The peak flux $F_{p}=F_{\nu, \max } \propto E_{\mathrm{K}}$ is bright: typically $10-100 \mathrm{mJy}$. The VLA (the vertical solid blue line) can easily detect the transients.

The dashed-dotted black lines indicate the distribution for radio $(150 \mathrm{MHz})$ transients. As we have discussed, this lowfrequency emission is suppressed by the self-absorption, and jet break is likely to happen before it becomes optically thin. The peak time of the light curve is determined by the jet break time $t_{p} \propto E_{\mathrm{K}}^{1 / 3} \theta_{j}^{8 / 3}$. For the fixed $\theta_{j}=20^{\circ}$, we find that the peaktime distribution is similar to that for $10 \mathrm{GHz}$ transients and it peaks around $t_{p} \sim 10$ days. However, since the emission is still suppressed by the self-absorption at the peak time, the peak flux is much lower: $F_{p} \sim 0.1 \mathrm{mJy}$. Approximately $30 \%$ of the $150 \mathrm{MHz}$ transients are brighter than the sensitivity limit of 48 LOFAR stations (the vertical dashed black line), and all are brighter than the sensitivity limit for SKA1-Low (the vertical dashed-dotted black line).

Typical afterglow light curves for a selection of on-axis orphan afterglows are shown in Figure 3. An average luminosity distance of $\sim 220 \mathrm{Mpc}$ for NS-NS GW detectable mergers from our sample is used. X-ray, optical, and radio (10 $\mathrm{GHz}$ ) are shown for four combinations of $\Gamma$ and $E_{\mathrm{K}}$. The vertical dashed line in each panel represents the deceleration time $t_{\mathrm{dec}}$, as $t_{\mathrm{dec}}$ is most sensitive to $\Gamma$ (see Equation (3)); the lower Lorentz factor cases (top two panels) have a significantly later deceleration time. The vertical dotted line in each panel represents the jet break time $t_{j}$, a jet half-opening angle $\theta_{j}=20^{\circ}$ is used throughout, for narrower(wider) jet halfopening angles the break time will be at earlier(later) times. The jet break time is only weakly dependent on the kinetic energy (see Equation (7)). In all cases the X-ray (green dasheddotted line) and the optical (thin red line) peak at the deceleration time; the $10 \mathrm{GHz}$ (thick blue line) is shown to peak at a later time $t_{m}$ when the typical frequency $\nu_{m}(t)$ crosses the radio frequency. In all cases at times earlier than $t_{\mathrm{dec}}$ the flux is $\propto t^{3}$, for the X-ray and optical the flux at $t_{\mathrm{dec}}<t<t_{j}$ is $\propto t^{-3(p-1) / 4}$. At $10 \mathrm{GHz}$ the flux is $\propto t^{1 / 2}$ at $t_{\mathrm{dec}}<t<t_{m}$, and $t^{-3(p-1) / 4}$ after $t_{m}$ and before $t_{j}$. In all cases at $t>t_{j}$ the flux is $\propto t^{-p}$.

\section{EVENT RATES AND ON-AXIS PROBABILITY}

The Swift satellite has been detecting short GRBs at a rate of $\sim 10 \mathrm{yr}^{-1}$ since the launch in 2004 , and $\sim 1 / 4$ of the detected events have measured redshifts (Swift GRB catalog). Unfortunately no Swift short GRB with known redshift has been detected within the advanced LIGO/Virgo range for face-on NS-NS mergers $D \sim 300 \mathrm{Mpc}$, and only three (061201, 080905A, and 150101B) have occurred within the face-on NS-BH range $D \sim 600 \mathrm{Mpc}$ (Abadie et al. 2010). Metzger \& Berger (2012) estimate that $\lesssim 0.03$ (0.3) short GRBs per year, with redshift measurements, are currently being localized by Swift within $D \sim 300 \mathrm{Mpc}(600 \mathrm{Mpc})$. Considering that the field of view of the Swift BAT is $\sim 2$ sr, the all-sky rate of
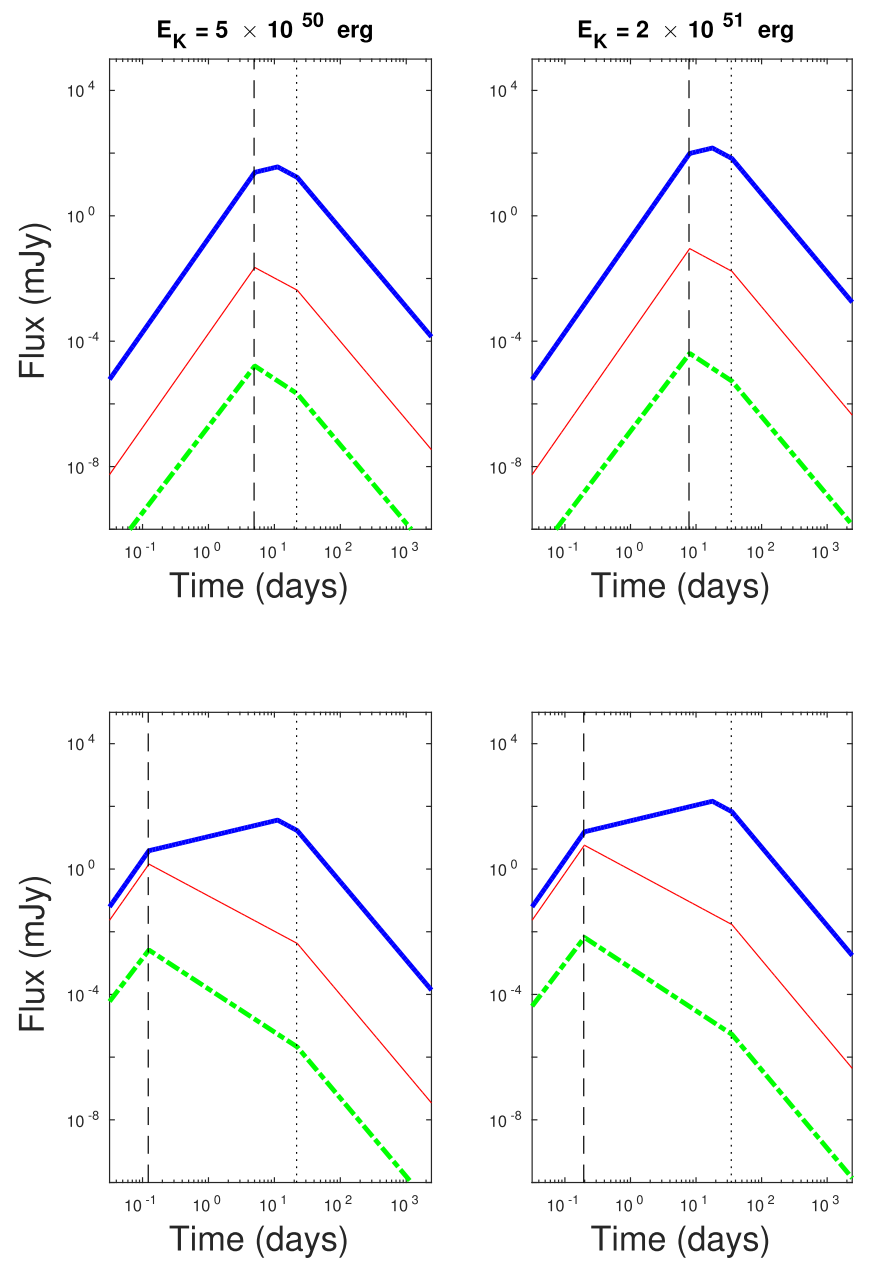

Figure 3. The light curves of on-axis orphan afterglows at $220 \mathrm{Mpc}$ with various bulk Lorentz factor $\Gamma$ and isotropic kinetic energy $E_{\mathrm{K}}$. The top(bottom) two panels have $\Gamma=5(20)$, and the left(right) panels have an energy $E_{\mathrm{K}}=0.5(2) \times 10^{51} \mathrm{erg}$. X-ray afterglow are shown as dashed green lines, optical are shown as red thin solid lines, and radio $(10 \mathrm{GHz})$ are shown as blue thick solid lines. The vertical black dotted lines represent the deceleration time $t_{\mathrm{dec}}$ and the jet-break time $t_{j}$ (assuming a $\theta_{j}=20^{\circ}$ )

detectable short GRBs with or without redshift information is higher by a factor of $\sim 25$.

If the distribution of $\Gamma$ is described by the power-law $N(\Gamma) \propto \Gamma^{-a}$, when we consider the rate of jets from mergers regardless of inclination or detectability, the rate for failed GRBs would be higher than the short GRB rate. For a local population $D \lesssim 300 \mathrm{Mpc}$, we find that the fraction of failed events is about $66 \%$ for $a=1.5,78 \%$ for 1.75 , and $87 \%$ for 2 (the same rates are obtained for a population of $D \lesssim 600 \mathrm{Mpc}$ ). If $a=1.75(2)$, the failed GRB rate is higher by a factor of $\sim 3.5$ (6.7) than the short GRB rate (i.e., the ratio of failed to successful GRBs). The all-sky rate of the failed GRBs with or without redshift information is about 2.6(5.1) per year for the NS-NS range and 26(51) per year for the NS-BH range. Here we assumed the jet opening angle distribution does not depend on the Lorentz factor of the jets (i.e., GRBs and failed GRB jets have the same opening angle).

The jet half-opening angle is not well constrained for short GRB jets (the median value for 248 long GRBs is $\theta_{j} \sim 13^{\circ}$; Fong et al. 2015). Using four short GRBs that have temporal steepenings on a timescale of $\sim 2-5$ days, the median value is estimated as $\sim 6^{\circ}$ (Fong et al. 2015). However, the majority of 


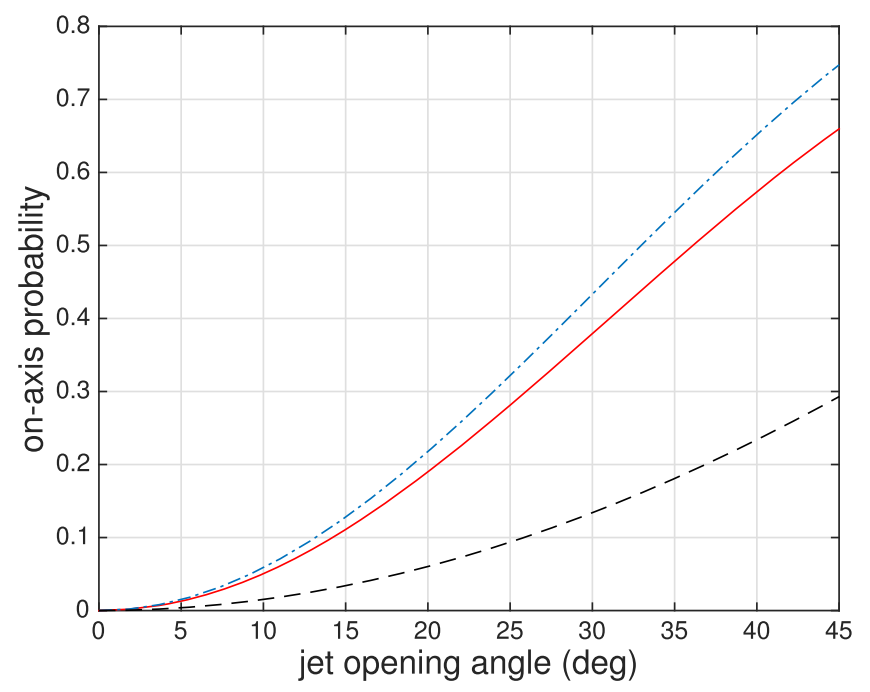

Figure 4. On-axis probability as a function of a jet half-opening angle $\theta_{j}$. The beaming factor $f_{b}=1-\cos \theta_{j}$ (black dashed line), the simple approximation $A^{3} f_{b}$ (blue dash-dot line), and the Monte Carlo results (red solid line).

short GRBs do not have detected jet break; the inclusion of these bursts is essential in understanding the true opening angle distribution. Based on a probability argument, Fong et al. (2015) obtain the median value $\theta_{j} \sim 16^{\circ}$ and $33^{\circ}$ if the maximum possible angle is $30^{\circ}$ and $90^{\circ}$, respectively.

If the typical jet half-opening angle of short GRBs is $\theta_{j} \sim 16^{\circ}$, the beaming factor is $f_{b} \equiv 1-\mu \sim 4 \times 10^{-2}$ where $\mu=\cos \theta_{j}$, and only a small fraction of short GRB jets point toward us (see the black dashed line in Figure 4). However, since the GW polarization components $h_{+} \propto\left(1+\cos ^{2} i\right)$ and $h_{\times} \propto 2 \cos i$ depend on the inclination angle $i$ of the binary, mergers emit GWs much more strongly along the polar axis than in the orbital plane. Considering that the jets from the mergers are also likely to be directed along the polar axis, Kochanek \& Piran (1993) show that when a GRB is associated, the $\mathrm{GW}$ amplitude $h$ is stronger by a factor of $A \equiv\left(1+11 \mu / 16+11 \mu^{2} / 16+\mu^{3} / 16+\mu^{4} / 16\right)^{1 / 2}$ than the amplitude averaged over the sky (as seen from the source). The distances out to which GW detectors could detect the binary increases by a factor of $A$ if the jet points toward us (we define an on-axis event as any jet where the inclination is within the half-opening angle, $i \leqslant \theta_{j}$ ).

When we consider a sample of merger GW events detected by a GW detector with sensitivity $h_{c}$, their jets would tend to be directed to us. This is because on-axis events are detectable at a larger distance. The on-axis probability could be higher by roughly the volume factor of $A^{3}$ (the blue dashed-dotted line, Figure 4 ) than the simple geometric estimate $f_{b}$ (i.e., our lineof-sight falls within the opening angle of the jet with a higher probability). We also conduct a Monte Carlo simulation to estimate the on-axis probability. In the simulation, mergers are uniformly distributed in space, with a random inclination angle, and they emit GWs with amplitude $h \propto \sqrt{h_{+}^{2}+h_{\times}^{2}} / D$. After selecting the events detectable by a GW detector: $h>h_{c}$, we evaluate the fraction of the events which have an inclination

\footnotetext{
1 If the property of the jet depends on the angle $\theta$ from the jet symmetry axis (e.g., $\Gamma \propto \theta^{-b}$ outside of some core angle), only the central part could have Lorentz factors high enough to produce $\gamma$-rays. Although a detailed study is beyond the scope of this paper, the failed GRB rate could be even higher for structured jets.
}

angle smaller than a given jet half-opening angle $\theta_{j}$; we assume uniform jets with a top-hat distribution throughout. ${ }^{1}$ The result (the red solid line) does not depend on the detector sensitivity as long as the merger distribution is homogeneous. If we consider GW trigger events, the on-axis probability (the red solid line; $13 \%$ and $44 \%$ for $\theta_{j}=16^{\circ}$ and $33^{\circ}$, respectively) is much higher than the beaming factor (the black dashed line). Although isotropic EM counterparts such as macronovae could be ideal to localize a large sample of GW events, $>20 \%$ of GW events would still be associated with the on-axis orphan afterglow of failed GRBs, especially when they have wider jet opening angles compared to short GRB jets. For long GRB jets, observational results indicate such a correlation $\Gamma \propto \theta_{j}^{-\kappa}$ with $0.3 \leqslant \kappa \leqslant 2.7$ (Kobayashi et al. 2002; Panaitescu \& Kumar 2002; Salmonson \& Galama 2002; Ghirlanda et al. 2013). The failed GRB rates could be higher than those discussed at the beginning of this section.

\section{CONCLUSIONS}

We have shown that failed GRBs are much more frequent than short GRBs when the Lorentz factors of jets from compact stellar mergers follow a similar power-law distribution as those observed for AGNs. For most events the internal dissipation process happens when the jet is still optically thick, and the photons produced by the dissipation process will be converted back to the kinetic energy of the jet. By using a simple Monte Carlo model, we have shown that even for the local merger population within the LIGO/Virgo range, the $\gamma$-ray emission from jets with $\Gamma \lesssim 30$ will not be detected by $\gamma$-ray satellites (e.g., Swift). For a power-law distribution of the jet Lorentz factors $N(\Gamma) \propto \Gamma^{-1.75}, 78 \%$ of compact object mergers that have jets result in a failed GRB. The failed GRB events will produce on-axis orphan afterglows at late times. Using the local short GRB rate as normalization, the all-sky rate of the on-axis orphan afterglow is about 2.6 and 26 per year for the NS-NS range $(300 \mathrm{Mpc})$ and $\mathrm{NS}-\mathrm{BH}$ range $(600 \mathrm{Mpc})$, respectively. The opening angle of jets for long GRBs was found to be a function of $\Gamma$ (e.g., Ghirlanda et al. 2013); if low- $\Gamma$ jets from compact-binary mergers have wider half-opening angles $\theta_{j}$ than those of short GRBs then the real rate would be higher than these.

We have evaluated the peak time and peak luminosity of the on-axis orphan afterglows in the X-ray, optical, and radio bands. However, it is usually difficult to model observational data for orphan afterglow candidates when the explosion time is unknown (i.e., the $t_{0}$ issue). For GW trigger events, GW signals will provide the explosion time $t_{0}$. The peak time distribution in the X-ray and optical band is rather wide, 0.1-10 days after the GW signals. Although the sky localization of sources by GW detectors is not accurate enough for follow-up observations by most conventional telescopes (Abbott et al. 2016a), $85 \%$ of the on-axis orphan afterglows are brighter than $m_{g}=21$. The current and upcoming optical transient search (e.g., iPTF/ZTF, Pan-STARRS, GOTO, BlackGEM, Kiso, SkyMapper, Subaru HSC, LSST) should be able to detect the optical transients. The X-ray and/or optical detection can be followed by radio observations (e.g., VLA); also several radio instruments have the potential to be leading transient detectors due to their large fieldof view (e.g., SKA, LOFAR, APERTIF, MWA). Radio emission is expected to peak around 10 days after the merger events. Optical and 
radio observations will constrain the opening angle of low- $\Gamma$ jets (and high $\Gamma$-jets).

Since merger jets from GW trigger events tend to be directed to us, the on-axis probability (e.g., $13 \%$ and $44 \%$ for $\theta_{j}=16^{\circ}$ and $33^{\circ}$, respectively) is much higher than the beaming factor $f_{b}=1-\cos \theta_{j}$. A significant fraction of GW events could be associated with on-axis orphan afterglows. Observations of onaxis orphan afterglows and GRB afterglows will enable us to determine the $\Gamma$ distribution of jets (e.g., clustered at high- $\Gamma$, a power-law distribution, a log-normal, or multiple populations), and it will provide constraints on the acceleration process of relativistic jets.

We thank the anonymous referee and Phil James for their constructive comments. This research was supported by STFC grants.

\section{REFERENCES}

Abadie, J., Abbott, B. P., Abbott, R., et al. 2010, CQGra, 27, 173001 Abbott, B. P., Abbott, R., Abbott, T. D., et al. 2016a, LRR, 19, 1 Abbott, B. P., Abbott, R., Abbott, T. D., et al. 2016b, PhRvL, 116, 06112 Band, D. L. 2006, ApJ, 644, 378

Beloborodov, A. M. 2011, ApJ, 737, 68

Berger, E. 2014, ARA\&A, 52, 43

Bloom, J. S., Giannios, D., Metzger, B. D., et al. 2011, Sci, 333, 203

Burrows, D. N., Kennea, J. A., Ghisellini, G., et al. 2011, Natur, 476, 421

Cenko, S. B., Krimm, H. A., \& Horesh, A. 2012, ApJ, 753, 77

Cenko, S. B., Kulkarni, S. R., Horesh, A., et al. 2013, ApJ, 769, 130

Cenko, S. B., Urban, A. L., Perley, D. A., et al. 2015, ApJ, 803, 24

Connaughton, V., Burns, E., Goldstein, A., et al. 2016, ApJ, 826, 6

Daigne, F., Bosnjak, Z., \& Dubus, G. 2011, A\&A, 526, 13

Dermer, C. D., Chiang, J., \& Mitman, K. E. 2000, ApJ, 537, 785

Fong, W., Berger, E., Margutti, R., \& Zauderer, B. A. 2015, ApJ, 815, 102

Gao, H., Ding, X., Wu, X. F., Zhang, B., \& Dai, Z. G. 2013, ApJ, 771, 86

Ghirlanda, G., Ghisellini, G., Salvaterra, R., et al. 2013, MNRAS, 428, 1410

Ghirlanda, G., Nava, L., Ghisellini, G., Celotti, A., \& Firmani, C. 2009, A\&A, 496, 585

Ghirlanda, G., Nava, L., Ghisellini, G., et al. 2012, MNRAS, 420, 483

Götz, D., Laurent, P., Lebrun, F., Daigne, F., \& Bosnjak, Z. 2009, ApJL, 695, L208

Granot, J., \& Piran, T. 2012, MNRAS, 421, 570

Granot, J., \& Sari, R. 2002, ApJ, 568, 820

Gruber, D., Goldstein, A., Weller von Ahlefeld, V., et al. 2014, ApJS, 211, 27

Hascoët, R., Beloborodov, A., Daigne, F., \& Mochkovitch, R. 2014, ApJ, 782, 5

Huang, Y. F., Dai, Z. G., \& Lu, T. 2002, MNRAS, 332, 735

Jorstad, S. G., Marscher, A. P., Lister, M. L., et al. 2005, AJ, 130, 1418

Kisaka, S., Ioka, K., \& Takami, H. 2015, ApJ, 802, 119

Kobayashi, S., Piran, T., \& Sari, R. 1997, ApJ, 490, 92

Kobayashi, S., Piran, T., \& Sari, R. 1999, ApJ, 513, 669
Kobayashi, S., Ryde, F., \& MacFadyen, A. 2002, ApJ, 577, 302

Kobayashi, S., \& Sari, R. 2001, ApJ, 551, 934

Kobayashi, S., \& Zhang, B. 2003, ApJL, 582, L75

Kochanek, C., \& Piran, T. 1993, ApJL, 417, L17

Levan, A. J., Tanvir, N. R., Cenko, S. B., et al. 2011, Sci, 333, 199

Liang, E., Yi, S. X., Zhang, J., et al. 2010, ApJ, 725, 2209

Liodakis, I., \& Pavlidou, V. 2015, MNRAS, 451, 2434

Lister, M., Cohen, M. H., Homan, D. C., et al. 2009, ApJ, 138, 1874

Lister, M., \& Marscher, A. P. 1997, ApJ, 476, 572

Lithwick, Y., \& Sari, R. 2001, ApJ, 555, 540

Marscher, A. P. 2006a, in AIP Conf. Proc. 856, RELATIVISTIC JETS: The Common Physics of AGN, Microquasars, and Gamma-Ray Bursts, ed. P. H. Hughes \& J. N. Bregman, (Melville, NY: AIP), 1

Marscher, A. P. 2006b, in PoS, Proc. VI Microquasar Workshop: Microquasars and Beyond, 21

Mészáros, P., \& Rees, M. 1992, MNRAS, 258, 41

Mészáros, P., \& Rees, M. 1997, ApJ, 476, 232

Metzger, B. D., \& Berger, E. 2012, ApJ, 746, 48

Mundell, C., Kopac, D., Arnold, D. M., et al. 2013, Natur, 504, 119

Nakar, E. 2007, PhR, 442, 166

Nakar, E., \& Piran, T. 2002a, NewA, 8, 141

Nakar, E., \& Piran, T. 2002b, MNRAS, 330, 920

Nakar, E., \& Piran, T. 2011, Natur, 478, 82

Nemmen, R. S. 2012, Sci, 338, 1445

Paczyński, B. 1986, ApJL, 308, L43

Panaitescu, A., \& Kumar, P. 2002, ApJ, 571, 779

Pe'er, A., Meszaros, P., \& Rees, M. J. 2005, ApJ, 635, 476

Piner, B. G., Pushkarev, A. B., Kovalev, Y. Y., et al. 2012, ApJ, 758, 84

Piran, T. 1999, PhR, 314, 575

Piran, T. 2004, RvMP, 76, 1143

Piran, T., Shemi, A., \& Narayan, R. 1993, MNRAS, 263, 861

Rhoads, J. E. 2003, ApJ, 591, 1097

Saikia, P., Elmar, K., \& Falcke, H. 2016, MNRAS, 461, 297

Salmonson, J. D., \& Galama, T. J. 2002, ApJ, 569, 682

Sari, R., Narayan, R., \& Piran, T. 1996, ApJ, 473, 204

Sari, R., \& Piran, T. 1999, ApJ, 520, 641

Sari, R., Piran, T., \& Halpern, J. P. 1999, ApJL, 519, L17

Sari, R., Piran, T., \& Narayan, R. 1998, ApJL, 497, L17

Shemi, A., \& Piran, T. 1990, ApJL, 365, L55

Steele, I., Mundell, C. M., Smith, R. J., Kobayashi, S., \& Guidorzi, C. 2009 Natur, 462, 767

Tang, Q. W., Peng, F. K., Wang, X. Y., \& Tam, P. H. T. 2015, ApJ, 806, 194

Thomson, C. 2007, ApJ, 666, 1012

van Eerten, H. J., \& MacFadyen, A. I. 2012, ApJ, 751, 155

Wanderman, D., \& Piran, T. 2015, MNRAS, 448, 3026

Woosley, S. E., \& Bloom, J. S. 2006, ARA\&A, 44, 1

Yamazaki, R., Asano, K., \& Ohira, Y. 2016, PTEP, 2016, 051E01

Yonetoku, D., Murakami, T., Nakamura, T., et al. 2004, ApJ, 609, 935

Yonetoku, D., Murakami, T., Gunji, S., et al. 2011, ApJL, 743, L30

Yost, S., Harrison, F. A., Sari, R., \& Frail, D. A. 2003, ApJ, 597, 459

Zauderer, B. A., Berger, E., Soderberg, A. M., et al. 2011, Natur, 476, 425

Zhang, B., \& Mészáros, P. 2004, Int. Mod. Phys, 19, 2385

Zhang, F., \& Li, Y. 2012, ApJ, 750, 11 